\title{
TO STUDY IMPACT OF PREFABRICATION ON PROFITABILITY OVER TRADITIONAL CONSTRUCTION- A REVIEW
}

\author{
Nitesh j Ramchandani \\ M.E.Student (Construction \& Management), \\ Department of Civil Engineering D. Y. Patil Institute \\ of Engineering \& Technology, Ambi Pune
}

\begin{abstract}
Prefabrication helps in terms of its impact on environmental protection and widely regarded as a sustainable construction method.The goal of prefabrication is to offer a way to get a well-designed building that is at least roughly tailored to resident needs. It could be stated that prefabrication system in building construction have the most effect on time and cost. One important aspect of this is the influence of prefabrication on construction waste reduction and waste handling activities including waste sorting, reuse, recycle, disposal. In recent research this topic has failed to take in to account its character of the process of construction waste minimization; integrating all essential waste handling activities has never being achieved. This topic helps to propose the possible impact arising from the application of prefabrication technology on construction waste reduction and the subsequent waste handling activity. Prefabricated building and structure are mounted from uniform prefabricated three-dimensional units, providing strength, pre-set thermal properties of structures, dynamic stability, immutability of geometric dimension of prefabricated elements during their manufacture, transportation and installation in special and difficult conditions. The main object of this project is to identify new technologies or methodologies in construction industries and to suggest for improvement of industry and study on cost effectiveness of pre-cast concrete construction.
\end{abstract}

Keywords: Prefabrication, Industrialization, Modularization, Preassembly, waste reduction.

\section{INTRODUCTION}

\subsection{Impact of prefabrication}

Prefabrication is the Practice of assembling components of a structure in a factory or other Manufacturing site and transporting complete assemblies to the construction site where the structure is to be

\author{
Prof. Hemant Salunkhe \\ Faculty in Department of Civil Engineering \\ Department of Civil Engineering D. Y. Patil Institute \\ of Engineering \& Technology, Ambi Pune
}

located. The role of prefabrication in architecture has been lauded for its potential to increase productivity and efficiency while not sacrificing quality. The values of better, faster and cheaper are applicable to developed countries such as the U.S., Japan, and Europe, whose middle class continues to demand this equation in buildings that range from the remarkable to the prosaic. Developing countries, including China, India, Africa and many parts of South America, that are beginning to rely on prefabrication have the potential advantages of realizing housing quickly and affordably; however, greater reliance on manufactured production has possibly more disadvantages than advantages for these cultures. With prefabrication, improved working conditions would seem to be agreeable to everyone: instead of building in the weather, international fabricators supply controlled environments with ergonomically considered equipment and yet in many fabrication environments, reliance on minimal skills, and a disconnect with the community in which workers live, leaves little room for continued fostering of personal and collaborative skills, culture, tradition and community building.

Prefabrication to be used to create a bland, monotonous landscape is an issue that developed countries' construction professionals must grapple with. Countries such as India are undoubtedly suffering a greater banality in the built environment by embracing prefabrication. Prefabrication is touted as offering a more sustainable solution to building, but developing counties already rely on vernacular practices for design and construction that require relatively low life cycle energy.

1.1.1 Need of the Prefabrication in construction project

1) Prefabrication is one of the key elements of industrialization in construction.

2) Prefabricated construction methods are presenting a range of techniques to improve the building construction, quality and how to reduce 
the negative impact of building production on the environment.

\subsubsection{Problems of the Construction Industry}

The subject of construction as an environmentally unfriendly activity has become arguably redundant. The level of pollution generated by construction alone is overwhelming and has been worsened globally due to the rapid rate of urban development. The challenges faced by and imposed by the construction industry on the environment and human life have been identified as construction waste, GHG and carbon emissions, high energy and resource consumption rates, and the lack of technological advancement in the face of the fast-paced movement of other industrial sectors.

\subsubsection{Structures and Policies}

In the precast concrete industry, outlined some of the constraints of the construction industry. Chief among them were the economy of a nation, government and labour policies, and climatic conditions. The author stated that it was the response of the construction industry to theseconstraints that has set in which has guided the industry's path to efficiency and effectiveness. With the unpredictability of any nation's economy, its governing policies as well as the changing climatic conditions in any given period, the construction industry has had to, and still has to cope with fluctuating demands in its effort to maximize flexibility. Thus, the construction industry has grown to become a characteristically fragmented industry. More so, there is a deficient working relationship that exists among the various participants of the construction process. Each sector looks to meet its inherent needs, disregarding the need for harmony required to effectively implement a complete construction process. The longterm results are seen in the industry's enduring inadequacies, its increased difficulty in meeting the demands and specifications of more complex projects with the required standards and quality, and expectedly, high costs of construction.

\subsubsection{Lack of Research and Development}

Another issue of major import is the patent lack of research and development in the construction industry. Although there has been a significant improvement in this regard over the last few years, the attempts are at best few and far between. Only in more recent times with the growing urgency to 'curb the excesses' of the industry have there been noticeable tracks on the path to sustainability in construction. Specifically apropos construction methods, the transition to the postindustrial age seems to be a perpetual struggle, set back by the industry's outright preference for the customary methods
- the conventional construction systems - and it's rather slow acceptance of industrial or factory-based building systems. Unlike other industries of the economy that are quick to embrace technological advancements such as the manufacturing industry which has a constant influx of new products that improve productivity and product quality, the construction industry is set back by its snailpaced adoption of technology. As a result, where masscustomization of goods and services has enhanced and continuously so, the quality of work and life, especially from the beginning of the 21 stcentury till date, the construction industry seems to be experiencing regression

\subsubsection{Environmental Impacts}

As earlier stated, construction activities propagate environmental pollution which eventually causes climatic change that is fast becoming the bane of human existence. Overwhelming scientific evidence points to climate change as the gravest threat to humans by humans. Since the 18 th century, precisely 1750 , the concentration levels of GHG have increased considerably. The most notable of these atmospheric pollutants is carbon dioxide (CO2). From that time till the present, $\mathrm{CO} 2$ emissions from both the combustion of fossil fuels and the manufacture of cement, a prime construction material, have contributed to over $75 \%$ increase in atmospheric $\mathrm{CO} 2$. According to $\mathrm{CO} 2$ Data, $\mathrm{CO} 2$ concentrations have increased from the mean monthly value of 315.71 in 1958 to the most recently recorded value of 396.18; a rise that has resulted in global warming. Climate scientists have declared that there is very limited time - years and not decades to balance CO2 and other GHG (CO2now.org 2011). Hence, energy conservation has become a crucial factor in mitigating the consequential emission of carbon and GHG attributed to the buildings.

Referred to in terms of $\mathrm{CO} 2$, the energy and carbon emissions associated with a building's life cycle occur in three uniquely interdependent stages: construction, occupation and demolition. As long as there is production, the extraction, refinement, manufacture, transportation and eventual use of raw materials, expend energy and create environmental impacts. Despite the fact that these impacts are considered 'hidden 'or 'embodied' and are usually regarded as inconsequential to the overall amount of energy consumed during the design and construction of a building, the total embodied energy and carbon emissions are markedly influenced by the choice of construction materials and construction technology. The energy associated with construction waste is another area of grave concern. In order to address the problem of embodied energy, it is important that the efficiency of construction and use of buildings be revised 


\subsubsection{Waste}

Construction waste has been construed to be one of the major pollutants of the environment. Waste by definition is anything in excess of the minimum requirement of equipment, labour, time and materials essential for production, which should be eliminated for itslack of added value to the product in question waste is defined as the excess material resulting from human and industrial activities, with no additional worth. Another definition of waste is any substance which constitutes a scrap material or an effluent or other unwanted surplus, arising from the application of any process, [and is required] to be disposed of as being broken, worn out, contaminated or otherwise spoiled. More specifically, construction waste is defined as "the by-product generated and removed from construction, renovation and demolition workplaces or sites of building and civil engineering structure" identify two main classes of building construction waste: structure waste and finishing waste. Structure waste refers to waste generated during the course of construction, such as abandoned timber plates/pieces, reinforcement bars and concrete fragments. As such, the classes are concrete, mortar, roof tiles, sand lime bricks, piles, stone tablets and other fragments of wood and metal. Sources of waste are categorized under design inaccuracy, equipment handling error, material management, procurement and residual sources

The impact of construction waste on the environment is borne on a global scale. For instance, 17\% (70 million tonnes) of the total generated waste in the world per year is solely from construction and demolition operations, making the construction industry the highest producer of controlled waste in the world. This amount is estimated to be $24 \mathrm{~kg}$ of waste per week per UK resident, four times as much as that generated by household activities. Similarly, in Australia, $44 \%$ of the 14million tonnes of waste reported annually is contributed by its building construction industry.

In Wei (2006)'s extensive study on waste management, several causes of the high levels of waste encountered in the construction sector are analysed. One such cause is the poor attitude towards waste management which is blamed on the mind-set of operatives, for whom it is predetermined that waste generation is inevitable in construction, and minimization efforts are of non-priority. In addition, the cultural attitudes of the employees of any organization play a large role in their perception of waste management practices. Where such practices have been successfully implemented, there are higher chances of a positive attitude towards their constant implementation. On the other hand, the fear of diversity that has long held back the construction industry could be a major hindrance to the acceptance of new construction methods that will adopt waste management. This fear is particularly attributed to a severe lack of knowledge about the environment, although in Chan's (1998) opinion, the media's influence has bridged this gap. Another main cause of poor waste management is design changes, of which the belief is that constant changes in design details during construction due to insufficient knowledge, experience and miscommunication, play a major role in high levels of construction materials waste.

\subsection{Problem Statement}

In Conventional Approach the subject of construction as an environmentally unfriendly activity has become arguably redundant. The level of pollution generated by construction alone is overwhelming and has been worsened globally due to the rapid rate of urban development also lacking in dynamic nature for quantitatively evaluating the possible impacts arising from the application of prefabrication technology on construction waste reduction. New technologies or methodologies in the Construction are completely absent.

\subsection{Objectives}

1) To identify the possible impacts arising from the application of prefabrication technology on construction waste reduction.

2) To identify new technologies or methodologies in the Construction Industry that could require new training or up-skilling of the trades.

3) Correlation and regression analyses will be used to analyse the data.

4) To compare the cost \& duration of conventional building to the prefab building by analyses

\subsection{Aim}

1) To effect economy in cost

2) To improve in quality as the components can be manufactured under controlled conditions.

3) To speed up construction since no curing is necessary.

4) To use the materials which possess their innate characteristics like light weight, easy workability, thermal insulation and combustibility etc.

\subsection{Advantage of prefabrication}

1) Prefabricated components speed up construction time, resulting in lower labour costs;

2) Work is not affected by weather delays (related to excessive cold, heat, rain, snow, etc.); 
3) The mechanization used in prefabricated construction ensures precise conformity to building code standards and greater quality assurance;

4) There are less wasted materials than in site-built construction;

5) There is less theft of material/equipment (and less property damage due to vandalism)

6) Worker safety and comfort level are higher than in site-built construction;

7) Computerization of the production process permits a high degree of customization, at an affordable cost;

8) Quality control and factory sealing and design can ensure high energy efficiency;

9) Cost savings through prefabrication can reduce the income required to qualify for a high ratio mortgage by up to one third compared to a conventionally built home of the same size.

10) Self supporting readymade components are used so the need for formwork shuttering and scaffolding is greatly reduced.

11) Construction time is reduced and buildings are completed sooner allowing on earlier return of the capital invested.

12) On-site construction and congestion is minimized.

\subsection{Principles}

\subsubsection{Main Reasons To Choose Precast Construction}

\section{Method Over Conventional In Method}

a. Economy in large scale project with high degree of repetition in work construction.

b. Special requirement in finishing.

c. Consistency in structural quality control.

d. Fast speed of construction.

e. Constraints in availability of site resources(e.g. materials \& Laborites )

f. Other space \& environmental constraints.

g. Overall assessment of some or all of the above factors which points to the superiority of adopting precast construction over convention method.

h. The following details gives. The cost implications of precast construction \& conventional in situ method.

i. Large groups of buildings from the same type of prefabricated elements tend to look drab andmonotonous.

j. Local Jobs are last.

\subsection{Classification}

The Prefabrication is classified as follow from the view of degree of Precast construction.

1) Small prefabrication

2) Medium Prefabrication

3) Large Prefabrication

4) Cast in Site Prefabrication

5) Off-Site (or) factory Prefabrication

6) Open system of prefabrication

7) Closed system of prefabrication

8) Partial prefabrication

9) Total prefabrication

\subsubsection{Small Prefabrication}

The first 3 types are mainly classified according to their degree of precast. Elements using in that construction for eg.:brick is a small unit precast and used in building. This is called as small prefabrication. That the degree of precast element is very low.

\subsubsection{Medium Prefabrication}

Suppose the roofing systems and horizontal members are provided with pretested elements those construction are known as medium prefabricated construction here the degree of precast elements are moderate.

\subsubsection{Large Prefabrication}

In large prefabrication most of the members like wall panels, roofing / flooring Systems, beams and columns are prefabricated. Here degree of precast elements are high.

\subsubsection{Cast - In - Site Prefabrication: Off - Site}

\section{(Factory) Prefabrication}

One of the main factor which affect the factory prefabrication is transport.The width of mad walls, mode of transport, vehicles are the factors which prefabrication is to be done on site on factory.

\subsubsection{Open System Of Prefabrication}

In the total prefabrication systems, the space framers are casted as a single unit and erected at the site. The wall fitting and other fixing are done on site. This type of construction is known as open system of prefabrication.

\subsubsection{Closed System of Prefabrication}

In this system the whole things are casted with fixings and erected on their position. 


\subsubsection{Partial Prefabrication}

In this method of construction the building element (mostly horizontal) required are precast and then erected. Since the costing of horizontal elements (roof / floor) often take there time due to erection of from work the completion of the building is delayed and hence this method is restored. In most of the building sites this method is popular more. Son in industrial buildings where the elements have longer spans. Use of double tees, channel units, cored stabs, slabs, hyperboloid shall etc., are some of the horizontal elements.

\subsection{Prefabrication Elements}

1. Flooring / Roofing system.

2. Precast Beams

3. Precast Columns

4. Precast wall panels.

5. Precast Slabs.

\subsubsection{Flooring / Roofing System}

Depending upon the composition of units, precast flooring units could be homogeneous or nonhomogeneous.

1) Homogeneous floors could be solid slabs, cored slabs, ribbed or waffle slabs.

2) Non-homogeneous floors could be multilayered ones with combinations light weight concrete or reinforced pre stressed concrete with filled blocks. Depending upon the way, the loads are transferred the precast floors could be classified as one way or two way systems.

\section{One Way System}

One way system transfers loads to the supporting members in one direction only. The precast elements of this category are channel slabs, hollow core slabs, hollow blocks and hollow plank system, channels and tiles system, light weight cellular concrete slab etc.

\section{Two Way Systems}

Transfer loads in precast element under this system etc.

\subsubsection{Precast Concrete Beams And Girders}

There are different kinds of precast concrete beams and girders, which serve as load-bearing support. These includethe rectangular beam, L-shaped beam and inverted 'T'beam

Prefabricated buildings are produced and manufactured in a controlled environment with the latest manufacturing technologies. They were designed with such flexibility to be able to adjust with changes made by the owner of the building. Repeatable design is one of the key benefits of using prefabricated buildings. Because of the consistent production of this material, the factory engineers are allowed to continually monitor the production process andmake the necessary improvements whenever possible to be able to deliver the best quality for their products. The prefabricated building process usually starts with assembling of the steel, concrete and wood, or pure concrete frames. The machine-cut wood, steel, or concrete walls are usually added to the prefabricated buildings with exact design specifications. Then, the electrical wiring and the plumbing are installed before readying the unit for transport to the site. Sometimes, redundant quality controlsare being done to check and confirm any spot-on manufacturing defects before the material leaves the factory.

\subsection{Preparing Materials Onsite}

Multitasking is one of the skills used when preparing prefabricated materials onsite. Prefabricated buildings are being made in the factory, while the construction service professionals are waiting at the site to prepare the foundation. This is where you will receive the completed frames and units of prefabricated buildings. The land on site is being graded, where asthe cement foundation is poured onto the ground. Piers are also placed as needed to be able to give support to the completed prefabricated units.

\subsection{Prefabricated Building Assembly And Finishing}

Once all the units are completed, they are shipped to the site. There, the foundation is ready so that the prefabricated buildings are to be craned to be set into place. The unit will be connected to the foundation and to each other to be able to create a solid and durable building. Also, the utilities are to be connected and personally chosen exterior designs of finishes are being added upon erecting the units. Prefabricated building or volumetric modular construction refers to a method of building a structure off-site, rather than a description of the finished product. Prefab buildings are typically built 60 to 90 percent off-site in a three-dimensional form, designed to be constructed at one location, and then used by occupants at another. Modern, custom, multi-story, factory-built buildings with concrete and steel floors, brick exteriors, sheetrock interiors, windows, lighting, computer hook-ups, electrical service, plumbing, heating, air conditioning and restrooms can include everything you need and can be constructed in half the time of a site-built building. 


\subsection{Stages of Prefab Building}

1. Design approval by the end user and any regulating authorities

2. Assembly of module components in a controlled environment

3. Transportation of modules to a final destination

4. Erection of modules to form a finished building.

\subsection{Use of Prefabrication}

1) The most widely used from of prefabrication in building and civil engineering is the use of Prefabricated Concrete \& prefabricated steel sections in structures where a particulars part on form is repeated many line.

2) Pouring Concrete section in a factory brings the advantages of being able to re-use moulds and the concrete cab be mixed on the spot without having to be transported to and pumped wet on a congested construction site.

3) Prefabricating Steel sections reduces on site cutting and welding costs as well as the associated hazard

4) Prefabrication techniques are wood in the construction of apartment slacks and housing developments with repeated housing units.

5) The technique is also used in office blocks, warehouses and factory buildings.

6) Prefabricated Steel and glass sections are widely used for the exterior of large buildings.

7) Prefabricated bridge elements and systems offer bridge designers \& Contractors significant advantages in terms of construction time, safety environmental impact construct liability and cost.

8) Prefabrication can also help minimize the impact from bridge building.

9) Radio towers for mobile phone and other service often consist of multiple prefabricated sections.

10) Prefabricated has become widely used in the assembly of aircraft and space craft with component such as wings and fuselage sections after being manufactured in different countries or states from the final assembly site.

\section{METHODOLOGY}

\subsection{Methodology of the study}

A prefabricated structure is defined as a structure built through the associationor completion on site of several elements built in a factory or assembled on site. For example new Italian seismic legislation defines a prefabricated structure as being composed of elements in prestressed reinforced concrete, assembled on site or in dedicated factories with industrial processes and assembled on site using dry or wet structural assembly. The parts that comprise a prefabricated building can be divided as follows:

a. Main structural elements that have to resist stress deriving from its own weight, from loads they bear and stress transmitted from elements connected to them. They have to make the structure solid as a whole forming rigid floors as in the case of floors;

b. Secondary structural elements, with load bearing functions, not essential to the general stability of the building that should be able to resist actions with adequate safety (own weight and loads they bear).

\subsection{Fundamentals Of Prefabrication}

\subsubsection{Modularization}

Modularization is defined as the off-site construction of a whole system prior to its transportation to the site of construction. The modules may often be required to be broken down into smaller sizes for ease of transportation. Modularization usually involves more than one trade.

\subsubsection{Prefabrication}

This usually involves a single skill or trade and is generally defined as a production process, which normally takes place at a specialized factory where different materials are combined to form the component of an endproduct. As long as the component is manufactured at a factory and is not a whole system, it is regarded as prefabricated.

\subsubsection{Preassembly}

By definition, preassembly is the combination of various materials and prefabricated components at a separate facility before installation as a single unit. This installation is carried out similar to the process of modularization in which the manufactured components are assembled close to the site, followed by on-site installment. Commonly regarded as a combination of modularization and prefabrication, preassembly usually involves works form various crafts and parts of different systems. 


\subsubsection{Industrialization}

This term refers to an inclusion of all three aforementioned categories of offsite construction. Industrialization is based on the concept of manufacturing and is defined as the procurement of technology, equipment and facilities in order to increase productivity, reduce manual labour and improve production quality.

\subsection{Flow of the project-}

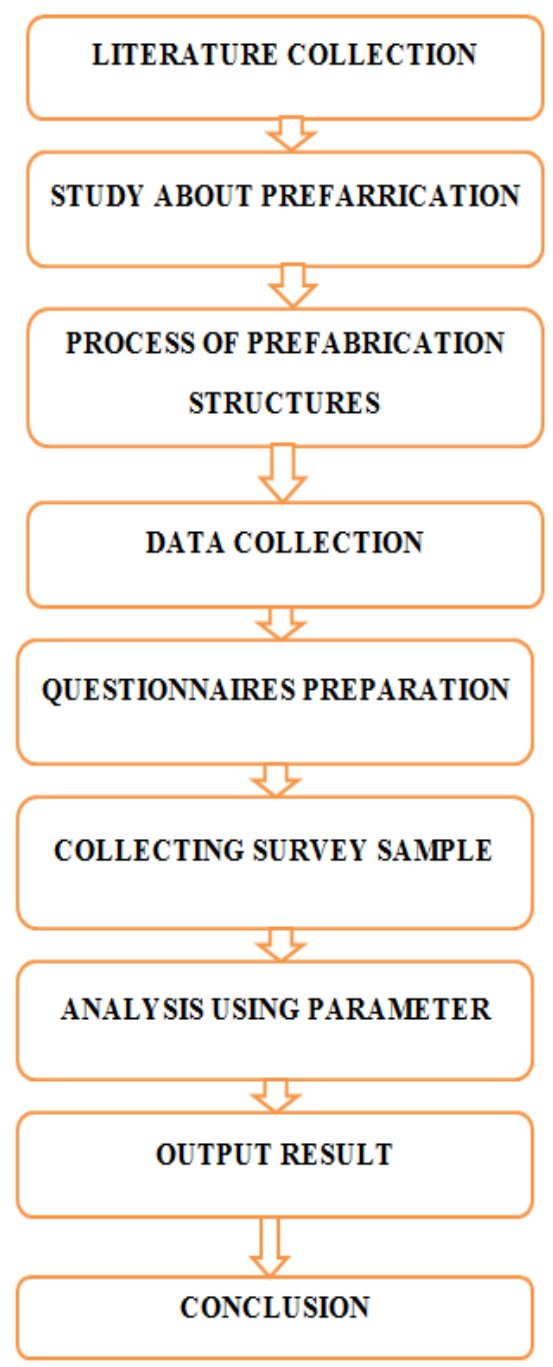

\subsection{Questionnaire Survey}

Questionnaire survey is made from different companies. From this we had known about the advantages and disadvantages of both conventional and prefabrication construction. And also from this we had known the present status and scope of the precast techniques. Survey respondents used a 5 point Likert scale to quantify their impact and ranking the score. The average amount of construction experience for all the respondents is 18 years. The low is 6 and the high is 60 . Most of the respondents have focused on Commercial and Residential construction in their careers. This chapter explores the data provided by the surveys in an in-depth manner. Each question is broken down and analyzed independently of the others. Then for each topic, the group of corresponding questions and their analyses are summarized.

\section{DATA COLLECTION}

\subsection{General}

The method of the study on comparison of prefabrication construction with conventional construction. A residential building is taken for comparing and it includes the preparation of plan, cost analysis, estimation of quantities, and determination of project duration.

\section{Estimation Of Quantities}

Estimation is used to find out the requirement of the materials for both the constructions. The details of the materials which are used in the construction from the companies were collected. By getting these details we can estimate the quantities of the materials.

\section{Project Duration}

Project duration of the each construction was collected from the similar companies and compares the time of completion period. It gives the project duration of precast and conventional construction of the building

3. Cost Analysis

This is the main factor which is considered in the project is to find out the comparison of cost analysis of double storey building for the prefab construction and conventional construction. In this analysis we want to consider the resources of labor, material and machineries. 
International Journal of Engineering Applied Sciences and Technology, 2019

Vol. 4, Issue 3, ISSN No. 2455-2143, Pages 533-540

Published Online July 2019 in IJEAST (http://www.ijeast.com)

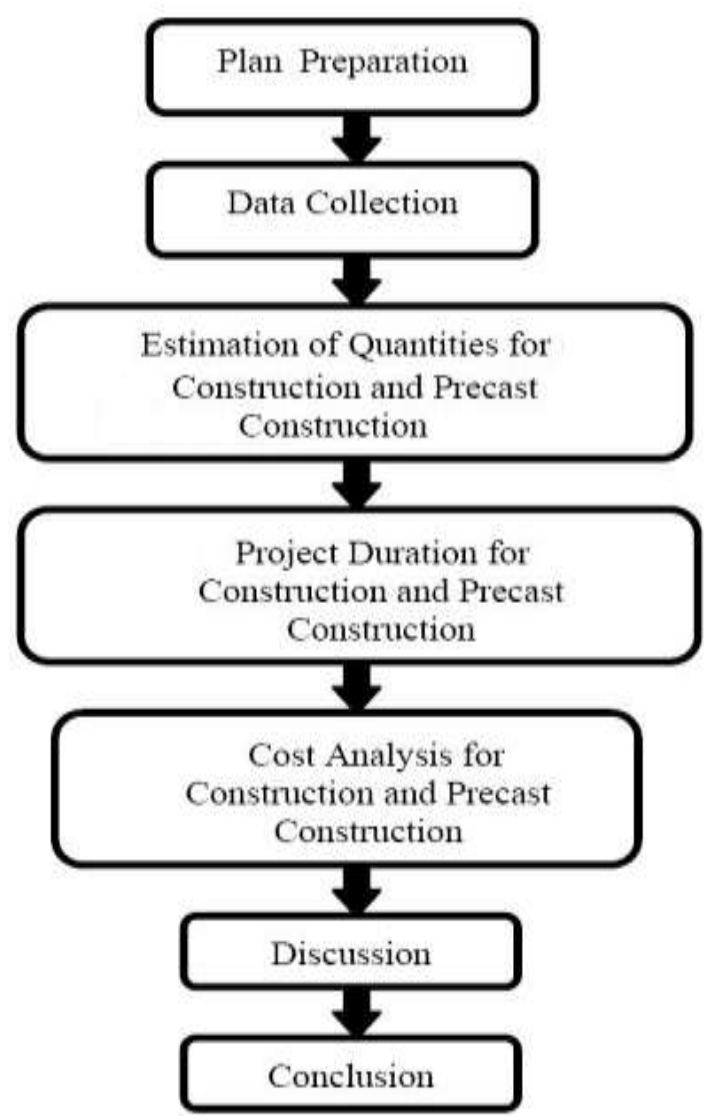

IV. CONCLUSION

Evidence from several studies showed that precast concrete construction, besides the improvement of a building's sustainable performance, include shortened construction time; overall reduced costs; enhanced quality and durability; improved health and safety, conservation of materials and energy; waste reduction; and finally reduced environmental emissions.

\section{REFERENCES}

(1)Abdallah A. (2007). Managerial and economic optimisations for prefabricated building systems.Technological and Economic Development of Economy. Vol. 13 (1), pp. 83-91.

(2)Abdou O.A., Murali K. \& Morsi A. (1996).Thermal performance evaluation of a prefabricated fiber-reinforced plastic building envelope system.Energy and Buildings (24), pp. 77-83.

(3)Abdulkadir M.R., Lee W.P., Jaafar M.S., Sapuan, S.M. \&Ali, A.A.A. (2006).Construction performance comparison between conventional and industrialised building systems in Malaysia. Structural Survey. Vol. 24 (5), pp. 412-424

(4)Andrews S., Prestressed Concrete (2003). Double tee panels [online]. [Accessed 10 December2011].

(5)Aye L., Ngo T., Crawford R.H., Gammampila R. \& Mendisa P. (2012).Life cycle greenhouse gas emissions and energy analysis of prefabricated reusable building modules.Energy and Buildings (47), pp. 159-168.

(6)Balaban O. (2012). The negative effects of construction boom on urban planning and environmentin Turkey: unraveling the role of the public sector. Habitat International (36), pp. 26-35.

(7)Baldwin A., Li H., Huang T., Kong C.W., Guo H.L., Chan N., and Wong J. (2009).Supporting pre-tender construction planning with virtual prototyping.Engineering, Construction and Architectural Management [online]. Vol. 16 (2), pp. 150-161.

(8)Dineshkumar N., Kathirvel P. "Comparative Study on Prefabrication construction with cast In-situ construction of residendal building" Vol.2 Issue 4, April 2015

(9)Alireza B.(2015) "Prefabrication system in Building construction" Vol 10, Number 24 pp 44258-44262.

(10) Asif M., Muneer T. \& Kelley R. (2010). Life cycle assessment: a case study of a dwelling home in Scotland. Building and Environment (42), pp. 1391-1394, 